\title{
Simulation Instructor Saved by Student
}

\section{To the Editor:}

B asic life support (BLS) is the first therapy in case of a cardiac arrest, and adequate performance is crucial for successful outcome. For this reason, it is increasingly recognized that every medical student should possess BLS skills to manage these life-threatening emergencies. ${ }^{1}$ In the educational program Technical Medicine in the Netherlands, all first-year students are therefore trained in BLS. The handson part of this training consists of multiple simulation sessions with lowfidelity manikins and is supervised by several undergraduate teaching assistants in close collaboration with professional instructors.

In preparation for the BLS course for the new first-year students, one of the student teaching assistants arrived for a meeting with the course coordinator to discuss and implement the new resuscitation guidelines. Within a few seconds after the student entered the teacher's room, the instructor (B.L., aged 29 years) collapsed at his desk and started gasping. At first, the student (B.H.) thought the instructor was simulating and wanted to test his BLS skills. After having said, "Are you joking me?" the student noticed the glassy eyes and concluded that this was neither a joke nor a test. His instructor was experiencing a real cardiac arrest! The student immediately called for help and started cardiopulmonary resuscitation, following the protocol he had learned during simulation sessions more than 2 years ago. When the university's in-house emergency service arrived with an automated external defibrillator, the instructor was found to be in ventricular fibrillation. After 6 minutes and 2 shocks, sinus rhythm was restored. After arrival in the hospital, no abnormalities were found; the teacher received an implantable cardiac defibrillator and fully recovered within a few days. Less than 2 weeks after the incident, the teacher went back to work and lectured about cardiovascular physiology as part of his regular course.

Thanks to timely and adequate handling of the student, the reanimation was very successful. It has already been shown that clinical experience alone is often insufficient to ensure the acquisition of basic clinical skills and that simulation-based education can dramatically improve adherence to American Heart Association guidelines in undergraduates. ${ }^{2}$ However, the interest of this particular case is that it shows the ability of a purely simulationtrained student, with no clinical experience regarding emergency situations, to be perfectly capable of performing BLS in real life. According to his own words, the technical part of the cardiopulmonary resuscitation was similar to the manikins used during simulationbased training. This shows that simulation can contribute to the acquisition of skills and that it can even, at least in BLS skills, replace clinical time, which is in contradiction with literature. ${ }^{3}$ The other remarkable fact is that the student was trained 2 years ago, whereas the retention of knowledge 6 months after training has been shown to decrease significantly without clinical re- inforcement. ${ }^{4}$ This obviously cannot be confirmed by this case. On the other hand, it must be considered that this particular student was trained to the level of instructor and, for this reason, was likely to be more skilled and confident than other students.

In conclusion, this unusual case presents a highly successful reanimation after sudden cardiac arrest, performed by a student without any clinical experience in life-threatening situations, who had undergone simulation-based training more than 2 years ago. This suggests a high effectiveness of simulation-based BLS education and underlines the paramount importance of medical student mastery of BLS skills in general.

Benno Lansdorp, MSc

Ben Hermans, BSc

Heleen Miedema, MSc

Faculty of Science and Technology

(B.L., B.H., H.M.), and MIRA-Institute for Biomedical Technology and Technical Medicine (B.L.)

University of Twente

Enschede, the Netherlands

\section{REFERENCES}

1. AAMC. The AAMC Project on the Clinical Education of Medical Students. Washington, DC: American Association of Medical Colleges; 2005.

2. Weller JM. Simulation in undergraduate medical education: bridging the gap between theory and practice. Medical Educ 2004; $38: 32-38$

3. Watson K, Wright A, Morris N, et al. Can simulation replace part of clinical time? Two parallel randomised controlled trials. Med Educ 2012;46:657-667.

4. Kaye W, Mancini ME. Retention of cardiopulmonary resuscitation skills by physicians, registered nurses, and the general public. Crit Care Med 1986; 14:620-622.
The authors declare no conflict of interest.

Reprints: Benno Lansdorp, MSc, Faculty of Science and

Technology, PO Box 217, 7500 AE Enschede, the

Netherlands (e-mail: b.lansdorp@utwente.nl)

Copyright (C) 2012 Society for Simulation in Healthcare ISSN: $1559-2332$

DOI: $10.1097 /$ SIH.0b013e31826f5ee1 